$\mathrm{J} 3 \mathrm{eA}$, Journal sur l'enseignement des sciences et technologies de l'information et des systèmes, Volume 4, Hors-Série 2, 7 (2005)

DOI : http://dx.doi.org/10.1051/bib-j3ea:2005707

(C) EDP Sciences, 2005

\title{
Étude numérique d'un moteur asynchrone
}

\author{
J. Lobry et A. Ishii
}

Service de Génie Électrique

Faculté Polytechnique de Mons

31 boulevard Dolez

B-7000 Mons, Belgique 


\title{
ETUDE NUMERIQUE D'UN MOTEUR ASYNCHRONE
}

\author{
J. Lobry* \& A. Ishii, Service de Génie Électrique, Faculté Polytechnique de Mons, 31, \\ Bd Dolez, B7000 Mons, Belgique, jacques.lobry@fpms.ac.be, aki.ishii@fpms.ac.be
}

\begin{abstract}
Résumé :
Cet article présente un projet de 50 heures destiné à des étudiants candidats ingénieurs de seconde année préparatoire. L'étude porte sur la modélisation numérique par éléments finis d'un moteur asynchrone de grande puissance. A partir de notions fondamentales acquises au cours d'Electricité Générale, les étudiants abordent de manière simple le principe de fonctionnement de la machine asynchrone. Ils découvrent ainsi les notions de champ tournant et de mise en mouvement induite. Cette dernière découle directement de la loi de Lenz et le calcul théorique du couple est déduit d'une analogie hydraulique. La simulation numérique est effectuée sous le logiciel FEMLAB®. Les étudiants doivent encoder la géométrie de la machine, les propriétés électriques des milieux et les courants dans les enroulements d'excitation. La résolution sous FEMLAB fournit des résultats sous la forme de cartes colorées et de lignes de champs à interpréter physiquement. La caractéristique mécanique est finalement déterminée par deux méthodes numériques et comparées aux résultats du constructeur.
\end{abstract}

Mots clés : Moteur asynchrone, Loi de Lenz, courants induits, champ magnétique, équations de Maxwell, substituts complexe, simulation numérique, éléments finis, FEMLAB.

\section{CONTEXTE}

La Faculté Polytechnique de Mons a récemment lancé une nouvelle initiative pédagogique qui concerne les étudiants candidats ingénieurs de seconde année préparatoire. Il s'agit de projets d'une durée de 50 heures réalisés en binômes et proposés par les sept Commissions de Diplômes de l'institution, de la Chimie à la Mécanique en passant évidemment par l'Electricité. Le projet est sanctionné par un rapport d'une vingtaine de pages noté par le tuteur et un rapporteur ainsi que par un exposé oral de quinze minutes présenté devant un jury constitué d'au moins trois personnes. C'est l'un des projets de la spécialité Electricité que nous présentons ici.

\section{DESCRIPTION GLOBALE DU PROJET}

Nous proposons l'étude numérique par éléments finis d'un moteur asynchrone de grande puissance dont les caractéristiques ont été fournies par un constructeur. Les étudiants concernés par le projet ne possèdent que des notions d'électricité générale et sont donc amenés à étudier le fonctionnement de la machine asynchrone sur la base de principes fondamentaux et par le biais d'analogies. Le traitement numérique par éléments finis suppose une modélisation issue des équations de Maxwell, notion normalement enseignée aux étudiants de seconde année. Le projet est ainsi une occasion de démythifier ce formalisme abstrait par une application concrète. L'analyse des résultats dérivés du calcul numérique permet aux étudiants de vérifier de nombreuses propriétés des grandeurs électromagnétiques telles l'induction magnétique, le champ magnétique et la densité de courant, l'effet de peau, la déformation des lignes de champ magnétique et les efforts qui en résultent. La finalité technique du projet réside dans la détermination de la caractéristique mécanique, soit le couple en fonction du glissement, de la machine. En second plan, le projet offre l'opportunité d'utiliser un logiciel de calcul par éléments finis et donc d'appliquer une technique numérique très répandue pour le traitement des problèmes d'électromagnétisme. Les étudiants sont sensibilisés aux divers paramètres qui influent sur la précision des résultats, notamment le caractère bidimensionnel de l'étude, l'hypothèse de linéarité des matériaux magnétiques ou encore la discrétisation géométrique adoptée.

\section{DEMARCHE PEDAGOGIQUE}

\subsection{Présentation du moteur asynchrone}

En premier lieu, le moteur asynchrone est présenté aux étudiants comme le moteur électrique le plus utilisé dans l'industrie. Son étude est ensuite entreprise de manière simple sur la base de principes fondamentaux et d'analogies bien ciblées. Pour ce faire, nous avons repris la présentation proposée dans [1] où l'auteur explique par la loi de Lenz le phénomène d'entraînement asynchrone d'un disque métallique soumis à l'influence d'un champ magnétique tournant créé par un aimant en rotation. Il reste alors à associer à cet exemple les notions de bobinage triphasé et de champ tournant. Les étudiants sont alors capables d'écrire les équations qui mènent à l'expression d'une onde tournante de champ magnétique. Les notions de pulsation synchrone $\omega$ et de glissement $g$ font naturellement leur apparition.

La même référence présente également une analogie hydraulique intéressante qui permet de déduire très simplement l'expression mathématique du couple 
asynchrone $\mathrm{C}$ en fonction des pertes par friction du système et de la vitesse de rotation. En généralisant, on obtient la formule de couple habituellement déduite d' un calcul laborieux de l'énergie magnétique de la machine asynchrone, soit :

$$
C=\frac{P_{J}}{g \omega}
$$

où $\mathrm{P}_{\mathrm{J}}$ représente les pertes Joule au rotor, $\mathrm{g} \omega$ la vitesse de glissement. Remarquons que cette expression est valable pour une machine bipolaire. Les étudiants sont invités à réfléchir au cas général de plusieurs paires de pôles.

L' approck théorique est bien entendu illustrée par une présentation des moteurs asynchrones de notre laboratoire. Les étudiants peuvent ainsi examiner de près la structure d' une machine électrique et les problèmes inhérents à sa construction.

\subsection{Aspect logiciel}

La partie essentielle du projet réside dans la simulation numérique par éléments finis du fonctionnement de régime d'une machine asynchrone. On invite donc les étudiants à acquérir les principes de base de la technique de calcul. Ils disposent pour cela d'une documentation suffisante [2,3]. Une fois les notions essentielles assimilées, les étudiants peuvent s'initier à l'utilisation d'un logiciel commercial. Nous avons retenu le logiciel FEMLAB, code de calcul par éléments finis multidisciplinaire opérant sous MATLAB ${ }$. Son caractère convivial et ses performances intéressantes en font un outil idéal pour une initiation à la CAO sur des dispositifs électrotechniques. Afin de permettre aux étudiants de se familiariser avec FEMLAB, nous les invitons à reproduire un exemple décrit dans le manuel d' utilisation. Ce premier contact leur permet de découvrir divers modes de représentation des grandeurs locales (induction, densité de courant, champ électrique, ...), d'effectuer le calcul de grandeur globale (couple, énergie, pertes Joule, ...) et d'examiner l'influence du maillage sur la précision des résultats.

\subsection{Description de la machine étudiée}

Le moteur étudié est une machine asynchrone de grande puissance dont une coupe est présentée à la figure 1 et dont les données constructives essentielles sont les suivantes :

- fréquence : $50 \mathrm{~Hz}$

- type d'alimentation : triphasée

- $\quad$ nombre de pôles : 4

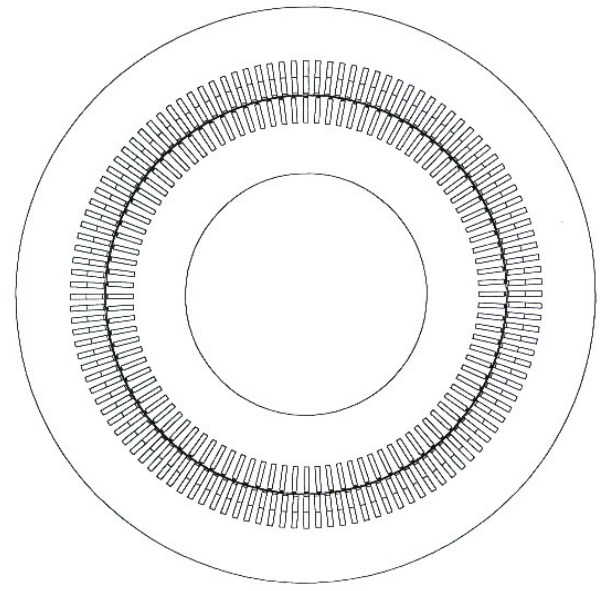

fig 1 : Coupe médiane de la machine étudiée

Grandeurs nominales sous glissement $g=1,5 \%$ :

- $\quad$ puissance à l'arbre $\mathrm{P}_{\text {méc }}=8280 \mathrm{~kW}$

- tension $\mathrm{U}_{\mathrm{n}}=6204 \mathrm{~V}$

- $\cos \bullet=0,932$

- rendement $\eta=0,95$

Stator :

- nombre d'encoches : 120

- pas : 1-25

- par pôle et phase : 10 encoches, 60 spires, 4 circuits en parallèle, enroulement double couche $2 \times 6$ fils

La répartition des courants dans les conducteurs est fournie aux étudiants.

Rotor :

- simple cage

- nombre d'encoches : 100

\subsection{Modélisation}

Ce paragraphe reprend la démarche de modélisation proposée aux étudiants. La discussion sur les différentes hypothèses est bien sûr ouverte.

Le calcul précis des efforts dans une machine tournante exige une simulation électromagnétique qui fournit la distribution du champ magnétique dans toute la structure ainsi que celle des courants induits au rotor. Le relevé de la caractéristique mécanique (couple - glissement) suppose des conditions de régime d'alimentation et une vitesse constante pour chaque point de calcul.

En premier lieu, une modélisation bidimensionnelle s'impose pour des raisons évidentes de commodité. Un modèle magnétodynamique en potentiel vecteur semble tout indiqué dans ce cas. Cependant, une étude rigoureuse nécessite la prise en compte de la saturation et des harmoniques d'espace, ce qui demande a priori un calcul pas à pas dans le temps, même si des courants sinusoïdaux sont injectés. D'autre part, la caractéristique mécanique est habituellement tracée sous tension d'alimentation 
constante, la prise en compte du circuit d'alimentation avec ses équations de Kirchhoff est donc également nécessaire. Une modélisation plus simple qui fournit malgré tout de bons résultats va être adoptée, elle est basée sur les hypothèses suivantes :

- les harmoniques d'espace sont négligés,

- la saturation est négligée (on adopte une perméabilité relative moyenne de 650),

- on relève la caractéristique mécanique à tension constante mais le courant est imposé à sa valeur effective pour chaque valeur du glissement.

La première hypothèse est importante car elle permet une étude en géométrie fixe sous excitation à la pulsation de glissement $g \omega$. L'ajout de la seconde restriction permet l'emploi des phaseurs de sorte que l'équation de base est :

$$
\nabla^{2} A-j g \omega \sigma \mu A=-\mu J_{s}
$$

où $\mathrm{A}$ est le potentiel vecteur, réduit à sa composante normale au plan d' étude, $\sigma$ est la conductibilité, $\mu$ est la perméabilité absolue et $\mathrm{J}_{\mathrm{s}}$ est la densité de courant d' exication.

Il s'agit ensuite d'effectuer successivement les opérations suivantes sous FEMLAB :

- introduire la géométrie de la machine en profitant des symétries,

- établir les conditions aux limites,

- fixer les propriétés physiques (conductibilité et perméabilité) et les termes sources : amplitudes et phases des courants statoriques, fréquence d'alimentation et glissement,

- effectuer un maillage,

- résoudre le système d'équations,

- afficher les cartes de champs (H, B, J).

Il apparaît que la machine peut être réduite à $1 / 4$ de sa géométrie en raison de la symétrique cyclique commune d'ordre 4 des stator et rotor ainsi que celle des courants d'excitation. Des conditions adéquates sur le potentiel vecteur doivent être imposées aux frontières de la cellule de symétrie traitée. Un fichier ". $d x f^{\prime \prime}$, réalisé sous le logiciel de dessin AutoCad ${ }^{\circledR}$, contient les données géométriques prêtes à l'exportation vers FEMLAB (figure 2).

\section{Calcul du couple}

Le couple sera calculé pour diverses valeurs du glissement sous la valeur effective du courant. Celui-ci se déduit des grandeurs locales fournies cidessus. Nous le déterminerons par la méthode du tenseur de Maxwell [4] dont une variante intéressante, proposée par Arkkio [5], est basée sur un calcul de moyenne opéré dans le domaine $\mathrm{D}_{\mathrm{e}}$ dé-

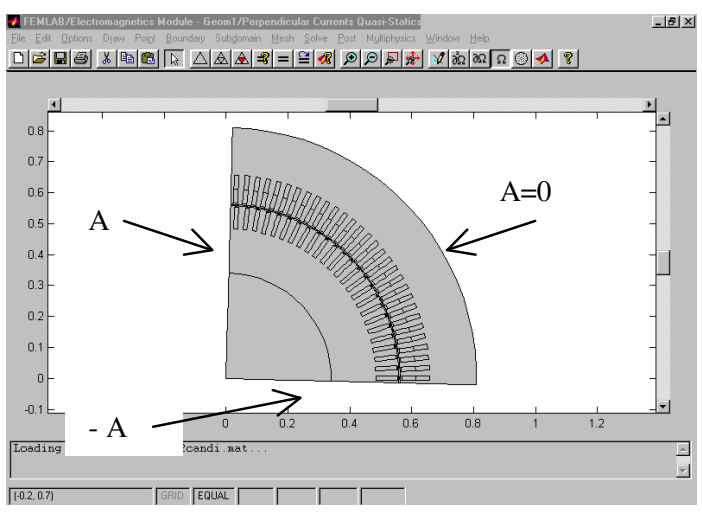

\section{fig 2: Géométrie traitée}

limité par l' entrefer géométrique, soit

$$
C=\frac{L}{\mu_{0}} \frac{1}{r_{s}-r_{r}} \iint_{D_{e}}(\mathbf{r} \times \mathbf{B})(\mathbf{B} . \mathbf{r}) \frac{1}{r} d S
$$

où $r_{r}$ et $r_{s}$ sont respectivement les rayons des rotor et stator au niveau de l'entrefer et $\mathbf{r}$ est le rayon vecteur issu de l'axe de ro tation.

Le couple ainsi obtenu est valable pour une longueur $\mathrm{L}$ de machine sans effets d' extrémité. Nous prendrons la longueur du stator majorée de $10 \%$ afin de tenir compte de ceux-ci.

\section{IMPLANTATION DU PROBLEME}

Après la mise à niveau théorique et la phase d'apprentissage sur FEMLAB, les étudiants entreprennent l'implantation du modèle numérique de la machine. Ils doivent établir en premier lieu la liste des données nécessaires: les conditions aux limites, les paramètres physiques des matériaux et la densité de courant injectée dans les conducteurs du stator. Cette dernière se déduit du calcul du courant I absorbé pour un glissement donné. L'évolution du rapport $\mathrm{I} / \mathrm{I}_{\mathrm{n}}$ en fonction du glissement est donnée par le constructeur. Il reste alors à calculer le courant nominal $I_{n}$ de la machine à partir des données fournies, soit :

$$
I_{n}=\frac{P_{m e ́ c} / \eta}{\sqrt{3} U_{n} \cos \varphi}
$$

On obtient ainsi la valeur de 864 ampères. La densité de courant est obtenue à partir des données sur le bobinage et la géométrie des encoches. Vu le modèle en nombres complexes, l'amplitude calculée doit être affectée de la phase adéquate $0, \pm 2 \pi / 3$ selon les conducteurs. La fréquence d'excitation est fixée à une série de valeurs liées aux glissements pour lesquels on souhaite calculer le couple.

Les conditions aux limites sont indiquées sur la figure 2. La condition $\mathrm{A}=0$ indique que le champ magnétique reste à l'intérieur de la machine, 
hypothèse classique généralement adoptée. La condition d'anti-symétrie sur les frontières internes est liée à la répartition des courants dans l'enroule ment d'excitation.

Le maillage généré par FEMLAB est repris à la figure 3. Il peut être orienté par l'ajustement de paramètres de manière à obtenir la finesse désirée dans les régions minces telles l'entrefer et de manière à assurer un compromis densité/précision.

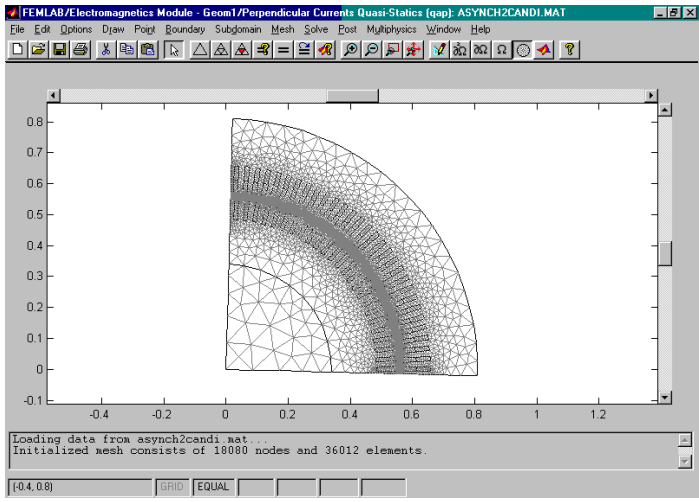

fig 3 : Maillage des sous-domaines

La résolution du système d'équations complexes fournit la liste des potentiels vecteurs en tous les noeuds de discrétisation. Il reste alors à tracer les cartes de champ magnétique et de densité de courant induite au rotor dont le but est dillustrer auprès des étudiants des propriétés essentielles : la conservation du flux magnétique, la réfraction des lignes de champ aux interfaces air-fer, l'effet de peau, ... . La figure 4 est représentative de ces observations.

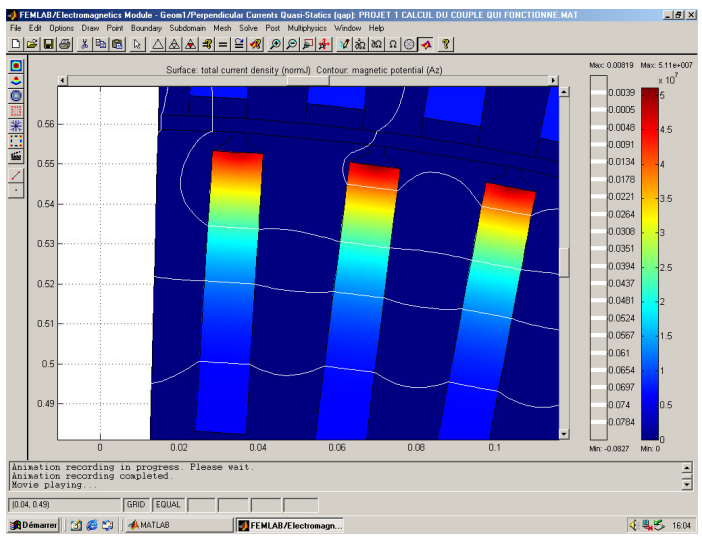

fig 4 : Lignes d'induction et courants induits $(g=1)$

Le couple se déduit de la relation d'Arkkio, que les étudiants encodent aisément dans la boîte de dialogue "subdomain integration ». Après une série de simulation pour différents glissement, on obtient la caractéristique de la figure 5 où l'on a également tracé celle du constructeur. Les étudiants constatent une bonne concordance entre les courbes excepté pour des glissements élevés en raison des effets de dispersion de flux plus marqués aux extrémités de la machine aux basses vitesses.

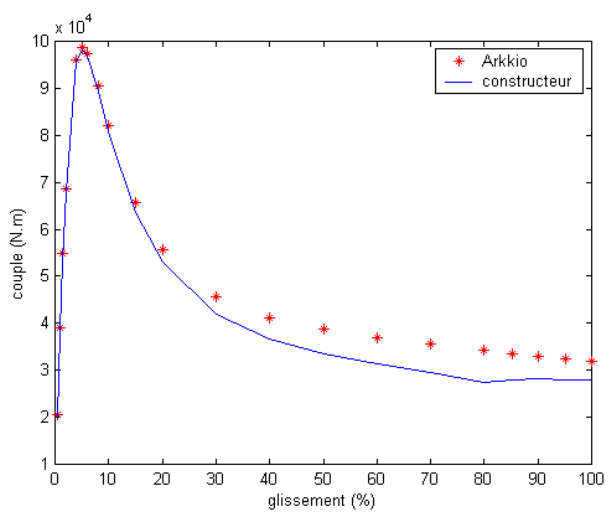

fig 5 : Caractéristique mécanique

\section{CONCLUSION}

Le projet a été une occasion de relier des concepts abstraits de l'électromagnétisme à une réalité tangible. Il est clair que l'outil logiciel a un impact important sur l'appréciation des étudiants. Les méthodes numériques de calcul de champs sont en effet une aide pédagogique précieuse dans l'ensei gnement des machines électriques. D'autre part, les objectifs généraux du projet ont été rencontrés : étude d'un problème concret, travail en groupe, respect d'une échéance, rédaction d'un rapport de synthèse et défense orale.

\section{Bibliographie}

1. M. Pouloujadoff, Machines asynchrones - Régime permanent, Techniques de l'Ingénieur, D3480.

2. M. Jufer \& J.C. Sabonnadière, Conception assistée par ordinateur - Moteur asynchrone, Techniques de l'Ingénieur, D3590.

3. T. Lecomte, Conception assistée par ordinateur Machine synchrone, Techniques de l'Ingénieur, D3595.

4. D.A. Lowther, P.P. Silvester, Computer-aided Design in Magnetics, Springer-Verlag, New York, 1986.

5. A. Arkkio, Finite element analysis of cage induction motors fed by static frequency converters, IEEE-MAG 26, No2, March 1990. 